\title{
Multiple small angle neutron scattering: A new two-dimensional ultrasmall angle neutron scattering technique
}

\author{
C. Grünzweig ${ }^{a)}$ \\ Paul Scherrer Institut, CH-5232 Villigen PSI, Switzerland \\ T. Hils \\ Physik Department E21, Technische Universität München, 85747 Garching, Germany \\ S. Mühlbauer \\ Physik Department E21, Technische Universität München, 85747 Garching, Germany \\ and Forschungsneutronenquelle Heinz Maier-Leibnitz (FRM II), 85747 Garching, Germany \\ M. Ay \\ Paul Scherrer Institut, CH-5232 Villigen PSI, Switzerland \\ K. Lorenz \\ Physik Department E21, Technische Universität München, 85747 Garching, Germany \\ R. Georgii \\ Physik Department E21, Technische Universität München, 85747 Garching, Germany \\ and Forschungsneutronenquelle Heinz Maier-Leibnitz, (FRM II), 85747 Garching, Germany \\ R. Gähler \\ Institut Laue Langevin, F38042 Grenoble, France \\ P. Böni \\ Physik Department E21, Technische Universität München, 85747 Garching, Germany
}

(Received 10 August 2007; accepted 19 October 2007; published online 12 November 2007)

\begin{abstract}
We report on the demonstration experiment of the multiple small angle neutron scattering (MSANS) technique at a $5.6 \mathrm{~m}$ long neutron beam line, leading to a $q$ resolution of $3 \times 10^{-4} \AA^{-1}$. The MSANS technique is based on two two-dimensional multihole apertures placed at the front end of the collimator and close to the sample, respectively. By choosing the proper MSANS geometry, individual diffraction patterns are superimposed leading to a large gain in intensity. Using MSANS as an option for standard small angle neutron scattering beam lines, the $q$ resolution could be increased to $10^{-5} \AA^{-1}$ without dramatically sacrificing intensity. (C) 2007 American Institute of Physics. [DOI: 10.1063/1.2809605]
\end{abstract}

Small angle neutron scattering (SANS) is a powerful technique for studying the structure of materials with lateral correlation lengths in the range of about 0.6 up to about $600 \mathrm{~nm}$. This corresponds to a $q$ range of $1-10^{-3} \AA^{-1}$. Measuring correlations in the micrometer range is a domain of light scattering; however, it often fails due to low contrast or opacity leading to multiple scattering in the sample. The research on biological samples, polymers, colloidal systems, cements, and microporous media are examples of a rising field, where the micrometer length scale plays an important role. Using the standard SANS technique for measurements of samples in the micrometer range leads to unacceptable losses in intensity - the increase in $q$ resolution by a factor of 10 in two dimensions leads to a drop in intensity by a factor of $10^{4}$. In the past and recent years, special ultrasmall angle neutron scattering (USANS) techniques have been developed. One technique is based on a double perfect crystal diffractometer in Bonse-Hart configuration. ${ }^{1-3}$ A second approach was realized by combining focusing mirrors with a standard SANS instrument. ${ }^{4,5}$ However, these two methods are sensitive to only one scattering direction and they are not compatible with sample environments as for standard SANS

\footnotetext{
${ }^{\text {a) }}$ Author to whom correspondence should be addressed. Electronic mail:
} christian.gruenzweig@psi.ch experiments. A third application is based on neutron spin echo as SANS instrument ${ }^{6}$ which is a real space approach. A fourth technique is using a multibeam collimator (array of aperture masks) ${ }^{7}$ however, the drawback of this technique is the low intensity, as cross talks between the holes of the aperture masks must be avoided. In this letter, we present the results of a new two-dimensional (2D) USANS technique named multiple small angle neutron scattering ${ }^{8}$ (MSANS) based on two multihole apertures. The experiment was performed at the $5.6 \mathrm{~m}$ long beam line MIRA at FRM II, where we succeeded to extend the $q$ range to $q=3 \times 10^{-4} \AA^{-1}$ using neutrons with a wavelength $\lambda=9.7 \AA$.

The setup of the MSANS experiment is illustrated in Fig. 1. It is based on two 2D multihole apertures which replace the collimator and sample apertures of a standard SANS instrument. $M_{e}$, the first aperture mask with lattice constant $a_{e}$ and hole diameter $d_{e}$ is installed at the front end of the collimator. $M_{s}$, the second aperture mask with lattice constant $a_{s}$ and hole diameter $d_{s}$ is placed at the distance $L_{1}$ behind $M_{e}$. The sample is placed directly behind $M_{s}$. The imaging detector with enhanced spatial resolution is located at the distance $L_{2}$ downstream of $M_{s}$. The basic idea behind the MSANS setup is superimposing the individual SANS patterns, which are well separated on the detector with the 


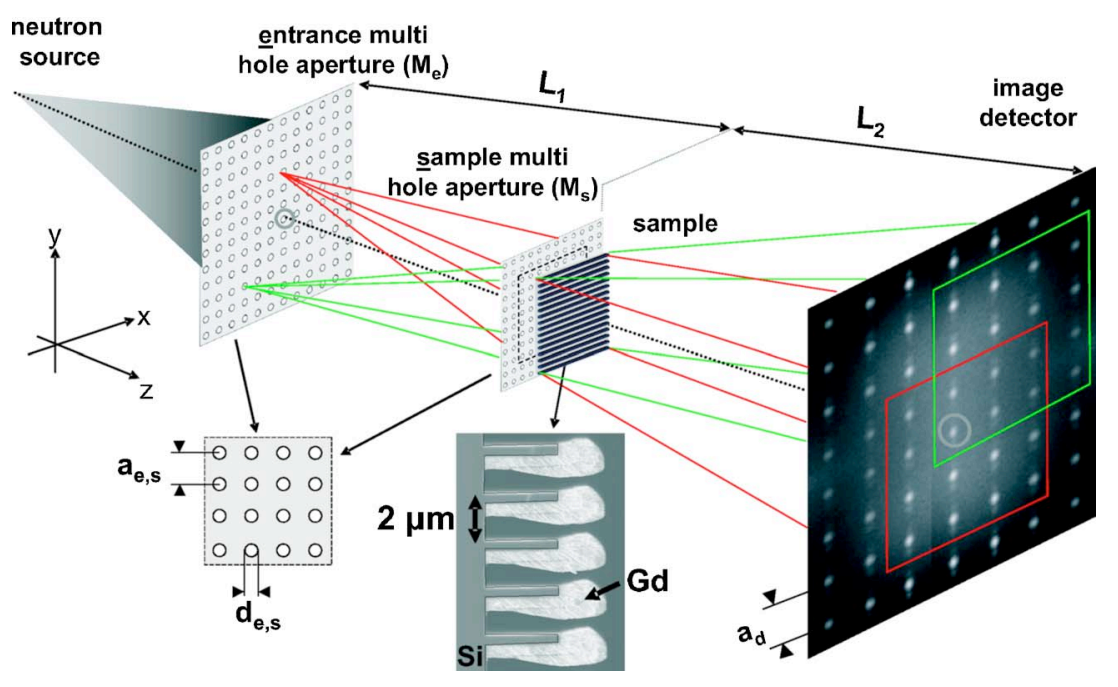

FIG. 1. (Color online) Schematic experimental setup of the MSANS option showing the $2 \mathrm{D}$ entrance multihole aperture $\left(M_{e}\right)$ with a lattice constant $a_{e}$ and hole diameter $d_{e}$, and the 2D sample multihole aperture $\left(M_{s}\right)$ with lattice constant $a_{s}$ and hole diameter $d_{s}$. Using appropriate values $a_{e, s}$, $L_{1}$, and $L_{2}$, the individual beams superimpose on the detector on a grid with the lattice constant $a_{d}$.

lattice constant $a_{d}$. This superposition is met if two conditions are fulfilled

$$
\begin{aligned}
& \frac{a_{d}}{a_{s}}=\frac{L_{1}+L_{2}}{L_{1}}, \\
& \frac{a_{d}}{a_{e}}=\frac{L_{2}}{L_{1}} .
\end{aligned}
$$

By choosing a one-to-one imaging system $\left(L_{1}=L_{2}\right)$, the lattice constants according to Eq. (1) are given by $a_{e}=2 a_{s}$ and $a_{d}=a_{e}$. For optimum performance, the hole diameters have chosen to be $d_{e} / d_{s}=a_{e} / a_{s}$, hence, $d_{e}=2 d_{s}$. The peak shape in the detector plane is the convolution of the object (circle of diameter $d_{e}$ ) with the point spread function of a pin hole camera, which in our case is a circle of diameter $d_{s} L_{1}$ $+L_{2} / L_{1}=d_{e}$ in the image plane. To first order, it may be approximated by a triangular function with a full width at half maximum (FWHM) $d_{d} \simeq L_{1}+L_{2} / L_{1} d_{\mathrm{s}}=d_{e}$. The intensity of a spot on the detector is proportional to the number of holes in mask $M_{e}$ seen from this detector spot through all holes in mask $M_{s}$, provided the beam divergence illuminates the setup sufficiently. The detector image (Fig. 1) consists of an inner area (overlap of green and red areas) with identical intensities ${ }^{9}$ and a penumbra where the intensities decrease linearly toward the border. For MSANS, the $q$ resolution is determined by the hole diameter $d_{e, s}$, while the $q$ range is determined by the lattice constant $a_{e, s}$. Both dimensions can be chosen individually.

For the experiment, the distances $L_{1}$ and $L_{2}$ were $2.6 \mathrm{~m}$. The hole diameters and the lattice constants were chosen to be $d_{s}=0.5 \mathrm{~mm}, d_{e}=1 \mathrm{~mm}, a_{s}=2.5 \mathrm{~mm}$, and $a_{e}=5 \mathrm{~mm}$, leading to spots on the detector with a FWHM of $d_{d}=1 \mathrm{~mm}$, separated by $a_{d}=5 \mathrm{~mm}$. The intensity of the neutrons was measured by means of a Mar345 image plate detector system with a nominal readout pixel size of $100 \mu \mathrm{m} . M_{e}$ and $M_{s}$ have sizes of $60 \times 60 \mathrm{~mm}^{2}$ and were fabricated from $1 \mathrm{~mm}$ thick cadmium plates having $11 \times 11$ and $21 \times 21$ holes, respectively. The sample, as sketched in Fig. 1, was a linear grating with a size of $32 \times 32 \mathrm{~mm}^{2}$. Figure 2 (a) shows the detector image of a gadolinium (Gd) absorption grating with a period of $2 \mu \mathrm{m}$, a duty cycle (DC) of 0.8 , and Gd thickness of $5 \mu \mathrm{m} .{ }^{10}$ The lines of the grating were placed horizontally resulting in diffraction spots along the vertical $y$ direction. The first column represents the area without sample. Extract- ing the profile of one of these spots (green box) along the $y$ axis leads to the green curve in Fig. 2(b) with a triangular shape as expected. The FWHM of $1 \pm 0.1 \mathrm{~mm}$ is in excellent agreement with $d_{e}=1 \mathrm{~mm}$. The orange curve in Fig. 2(b) shows the profile of a diffraction pattern [orange box in Fig. $2(a)]$ resulting from the Gd grating along the $y$ direction. The \pm 1 st order maximum appears at $q= \pm 3.2 \times 10^{-4} \AA^{-1}$, higher orders have very low intensity due to the large DC. The asymmetry in the intensity distribution is due to a small misalignment of the grating, namely, a tilt of the surface normal around the $x$ axis. Figure 2(c) shows the profile along the $y$ axis from adding all pictures in the detector plane. Figure 3(a) shows the detector image from a silicon phase grating with a period of $2 \mu \mathrm{m}$ and a DC of $0.5 .^{10}$ The depth of the

(a)

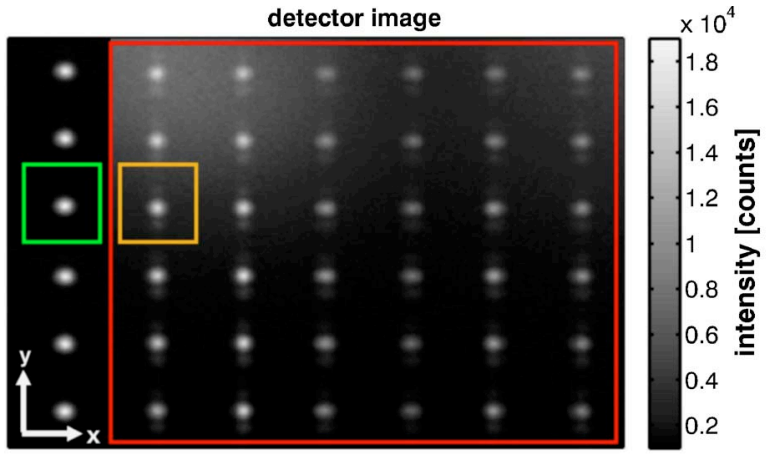

(b)

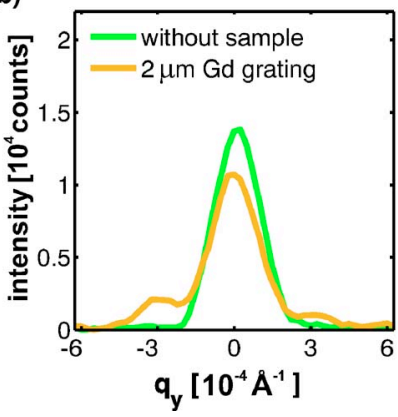

(c)

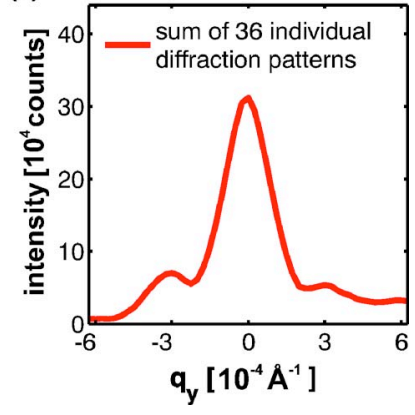

FIG. 2. (Color online) MSANS data from a $2 \mu \mathrm{m}$ period Gd absorption grating. (a) Detector image. (b) Section plots along the $y$ axis through one hole pattern without sample in the beam [green box in (a)] showing the intensity profile. The hole pattern with sample [orange box in (a)] showing the diffraction pattern of a one-dimensional (1D) Gd grating. The \pm 1 diffraction orders are clearly visible. (c) Section plot along the $y$ axis of 36 individual summed up diffraction patterns [red box in (a)]. 
(a)

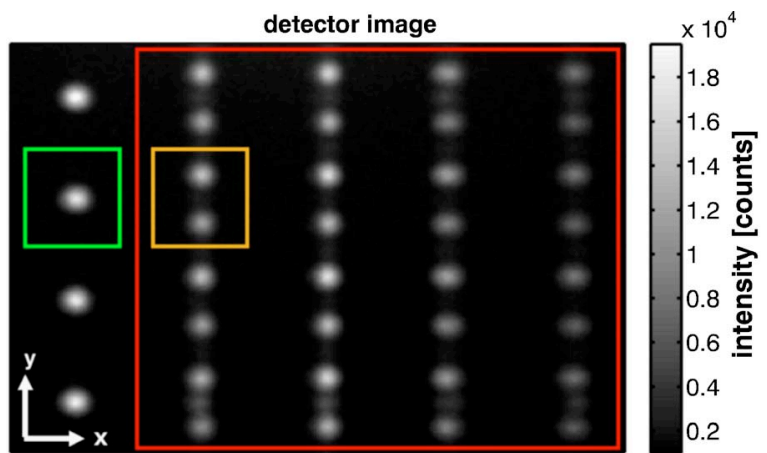

(b)

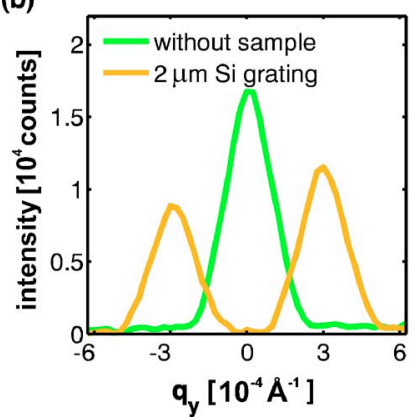

(c)

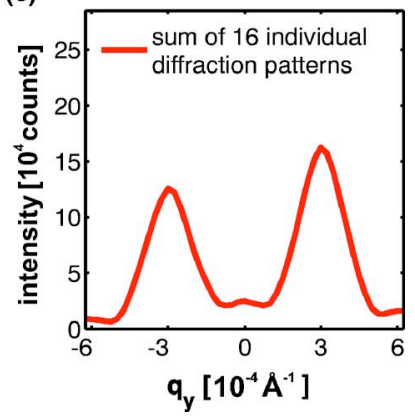

FIG. 3. (Color online) MSANS data of Si phase grating with a period of $2 \mu \mathrm{m}$. (a) Detector image. (b) Profiles along the $y$ axis through one hole pattern without sample in the beam [green box in (a)] showing the triangular intensity profile. The hole pattern with sample [orange box in (a)] showing the diffraction pattern of a 1D phase grating. (c) Section plot along the $y$ axis of 16 individual summed up diffraction patterns [red box in (a)].

grating was $15.5 \mu \mathrm{m}$ such that neutrons with $\lambda=9.7 \AA$ undergo a phase shift of $\pi$ when passing through the lines. The first column represents the area due to beams that do not intersect the sample. Figure 3(b) shows the profiles along the $y$ axis through a spot on the detector, as indicated by the orange and green box in Fig. 3(a) for beams intersecting and not-intersecting the grating, respectively. The disappearance of the direct beam demonstrates that the phase shift of $\pi$ was properly realized. Figure 3(c) shows the profile of the SANS pattern summed over the 16 individual spots within the area of the detector defined by the red box in Fig. 3(a). The shape of the curve is essentially conserved and the large gain in intensity is clearly visible. The central spot appears due to the contribution of neutron beams, which did not intersect the phase grating for a phase shift of $\pi$ properly.

In the following, we discuss the gain in intensity of the MSANS setup compared with a standard SANS instrument using a simple pinhole geometry. In the ideal case of MSANS, each beam emerging from the 121 holes $\left(d_{e}\right.$ $=1.0 \mathrm{~mm})$ of $M_{e}$ traverses one of the holes $\left(d_{s}=0.5 \mathrm{~mm}\right)$ of $M_{s}$ thus, producing 441 diffraction spots on the detector. Therefore, as compared to single apertures, the intensity is magnified by a factor $N=121 \times 441=53361$. The number of neutrons per second and per wavelength band $d \lambda$ at the sample position $d I_{\text {MSANS }} / d \lambda$ can be calculated from the spectral brightness $d^{2} \Phi / d \lambda d \Omega$ of the incoming guide, provided that the MSANS setup is fully illuminated

$$
\frac{d I_{\mathrm{MSANS}}}{d \lambda}=N\left(\frac{\pi d_{e} d_{s}}{4 L_{1}}\right)^{2} \frac{d^{2} \Phi}{d \lambda d \Omega} .
$$

For comparable $q$ resolutions of $\operatorname{MSANS}\left(L_{1}=L_{2}=2.6 \mathrm{~m}\right)$ and of a conventional SANS instrument $\left(L_{1}=L_{2}=20 \mathrm{~m}\right)$, the

single apertures $d_{e, s \text { (SANS) }}$ of the SANS instrument should be 7.69 and $3.85 \mathrm{~mm}$, respectively. ${ }^{11}$ With the parameters from above, the intensity gain of MSANS is $G=900$. Note that this gain is achieved with a much shorter instrument; however, the MSANS setup needs a bigger sample comparable to $M_{s}$ and has a much reduced $q$ range. However, by properly adjusting the lattice constants $a_{e, s}$ it is possible to match the $q$ range of MSANS to the $q$ resolution of the background SANS instrument thus, leaving no "hole" between both. Increasing the $q$ range by increasing the lattice constants of the masks will reduce the gain.

In summary, we have shown by using the MSANS technique that a $q$ resolution of already $3 \times 10^{-4} \AA^{-1}$ is obtained measured with a collimation length and detector distance of only $2.6 \mathrm{~m}$. The lack of intensity as expected for conventional SANS reaching this $q$ resolution was circumvent by superimposing individual SANS pattern leading to the corresponding gain of the MSANS option. By implementing the proposed technique at regular SANS beam lines, a $q$ resolution of the order of $10^{-5} \AA^{-1}$ can be easily achieved if proper care is taken with respect to gravitational effects on the neutrons by using lenses and correction prisms. As the MSANS setup is fully compatible with the regular SANS setup, experiments over a $q$ range of $10^{-5} \AA^{-1}<q<1 \AA^{-1}$ can now be readily performed without moving the sample from one beam line to another beam line.

The major advantage of MSANS as compared to the existing USANS instruments is the fact, that the scattering patterns can be collected in two dimensions in $q$ thus, reflecting the typically circular symmetry of diffraction patterns from homogeneous samples and the more involved diffraction patterns from anisotropic samples while maintaining an isotropic $q$ resolution.

This work was supported by the European Union within the Sixth Framework Program FP6 under Contract No. 505925.

${ }^{1}$ U. Bonse and M. Hart, Appl. Phys. Lett. 7, 238 (1965).

${ }^{2}$ M. Hainbuchner, M. Villa, G. Kroupa, G. Bruckner, M. Baron, H. Amenitsch, E. Seidl, and H. Rauch, J. Appl. Crystallogr. 33, 851 (2000). ${ }^{3}$ M. Strobl, W. Treimer, and A. Hilger, Appl. Phys. Lett. 85, 488 (2004). ${ }^{4}$ B. Alefeld, L. Dohmen, D. Richter, and Th. Brückel, Physica B 283, 330 (2000).

${ }^{5}$ E. Kentzinger, L. Dohmen, B. Alefeld, U. Rücker, J. Stellbrink, A. Ioffe, D. Richter, and Th. Brückel, Physica B 350, e779 (2004).

${ }^{6}$ M. Th. Rekveldt, Nucl. Instrum. Methods Phys. Res. B 114, 366 (1996). ${ }^{7}$ S. Désert, V. Thévenot, J. Oberdisse, and A. Brûlet, J. Appl. Crystallogr. 40, s471 (2007).

${ }^{8}$ R. Gähler, B. Fak, R. Golub, T. Hils, S. Klimko, J. Lal, S. Prokudaylo, and C. Stadler, Proc. SPIE 4785, 153 (2002).

"Ideally, one peak of the "inner area" is formed by superimposing $k$ different beams, where $k=\min \left(N_{e}, N_{s}\right)$ is the minimum of the number of holes $N_{e}$ and $N_{s}$ of the entrance and the sample mask $\left(M_{s}\right)$, respectively. Therefore, the intensity of a spot on the detector depends strongly on the number of illuminated apertures of $M_{s}$, i.e., on the size of the sample.

${ }^{10}$ C. Grünzweig, Fabrication and Testing of Diffraction Gratings for Phase Contrast Imaging with Neutrons, Diploma Thesis, University of Tübingen, 2006.

${ }^{11}$ Scaling of the SANS apertures for divergence of MSANS: $d_{e, s(\text { SANS })}$ $=L_{1,2 \text { (SANS) }} / L_{1,2 \text { (MSANS) }} \cdot d_{e, s(\mathrm{MSANS})}$. 